Draft Version DeCEMBer 6, 2018

Typeset using IATEX RNAAS style in AASTeX62

\title{
DISCOVERY OF A HOT SYMBIOTIC STAR IN THE COLD ANTARCTIC SKY: SYMBIOTICS ARE OUTLIERS IN SKYMAPPER uvgriz PHOTOMETRY
}

\author{
Adrian B. Lucy, ${ }^{1, *}$ J. L. Sokoloski, ${ }^{1,2}$ N. E. Nuñez, ${ }^{3}$ C. Wolf,,${ }^{4,5}$ T. Bohlsen, ${ }^{6}$ and G. J. M. Luna ${ }^{7,8,9}$ \\ ${ }^{1}$ Columbia University, Dept. of Astronomy, 550 West 120th Street, New York, NY 10027, United States \\ ${ }^{2}$ Large Synoptic Survey Telescope Corporation, 933 North Cherry Ave, Tucson, AZ 85721, United States \\ ${ }^{3}$ Instituto de Ciencias Astronómicas, de la Tierra y del Espacio (ICATE-CONICET), \\ Av. España Sur 1512, J5402DSP, San Juan, Argentina \\ ${ }^{4}$ Research School of Astronomy and Astrophysics, Australian National University, \\ Canberra, ACT 2611, Australia \\ ${ }^{5}$ ARC Centre of Excellence for All-sky Astrophysics (CAASTRO) \\ ${ }^{6}$ Mirranook Observatory, Boorolong Rd, Armidale, NSW, 2350, Australia \\ ${ }^{7}$ CONICET-Universidad de Buenos Aires, Instituto de Astronomía y Física del Espacio (IAFE), \\ Av. Inte. Güiraldes 2620, C1428ZAA, Buenos Aires, Argentina \\ ${ }^{8}$ Universidad de Buenos Aires, Facultad de Ciencias Exactas y Naturales, Buenos Aires, Argentina \\ ${ }^{9}$ Universidad Nacional Arturo Jauretche, Av. Calchaqui 6200, F. Varela, Buenos Aires, Argentina
}

\section{A YELLOW SYMBIOTIC AT EXTREME DECLINATION}

Using near-ultraviolet flux excess and variability from the SkyMapper Southern Sky Survey ${ }^{1}$ as novel diagnostics to search for symbiotic stars - cool giants accreting onto compact objects, typically white dwarfs (WDs) -, we report that Hen 3-1768 (三ASAS J195948-8252.7) is a symbiotic star. It may be an optimal target for continuous monitoring by Antarctic telescopes; at a declination of $-82.877^{\circ}$, it is now the closest known symbiotic to either geographic pole, and the only known symbiotic more southern than the Small Magellanic Cloud (Belczyński et al. 2000; Akras et al. 2019, ApJS, in press). Figure 1 shows that Hen 3-1768 produced unambiguous emission from Raman O VI 6830,7088 He II $4686 \AA$, and other transitions, proving that it is a symbiotic star (e.g., Shore et al. 2014). Comparing to Pickles (1998) template spectra, we preliminarily constrained the giant donor's spectral type to between K4 and K7, making Hen 3-1768 one of the dozen or so yellow symbiotics with stellar-type infrared (IR) colors currently known (e.g., Baella et al. 2016). The 2MASS IR colors $(\mathrm{J}-\mathrm{H})_{0}=0.82$ and $\left(\mathrm{H}-\mathrm{K}_{s}\right)_{0}=0.21$ (Skrutskie et al. 2006; de-reddened by total Galactic extinction $^{2}$ ) are consistent with this conclusion (cf. figure 1 in Baella et al. 2016).

\section{A NEW WAY TO FIND SYMBIOTICS}

Figure 1 shows that known symbiotics are outliers from the locus of our red giant (RG) sample in SkyMapper uvgriz photometry. The sample contains $\sim 210,000$ likely RGs, mostly isolated, built with IR photometry cuts loosely adapted from Li et al. (2016) and Bochanski et al. (2014). The arrhythmic multi-epoch source catalog of SkyMapper DR1.1 contains color and variability information for $3 \times 10^{8}$ objects in a 20,200 $\mathrm{deg}^{2}$ footprint (Wolf et al. 2018). The uvgriz filters include an intermediate-band violet $(v)$ filter and a Strömgren-like near-ultraviolet $(u)$ filter. Known symbiotics exhibit excess $u$ flux relative to the $v$ and especially $g$ fluxes. Variability from the accreting WD can also lead some symbiotics to have larger-amplitude $u$ flux variability than otherwise-similar isolated RGs. The argument for symbiotic activity is further strengthened when the $z$ flux does not track with the $u$ flux. We prioritized Hen 3-1768 for spectroscopic follow-up because it met all these criteria independently.

We selected Hen 3-1768 as a candidate using only SkyMapper, 2MASS, and WISE, but additional evidence abounds. GAIA DR2 (Gaia Collaboration et al. 2018) and RAVE DR5 (Kunder et al. 2017) each identify Hen 3-1768 as an

Corresponding author: ABL

lucy@astro.columbia.edu

* LSSTC Data Science Fellow

1 http://skymapper.anu.edu.au

${ }^{2}$ https://irsa.ipac.caltech.edu/applications/DUST/ 

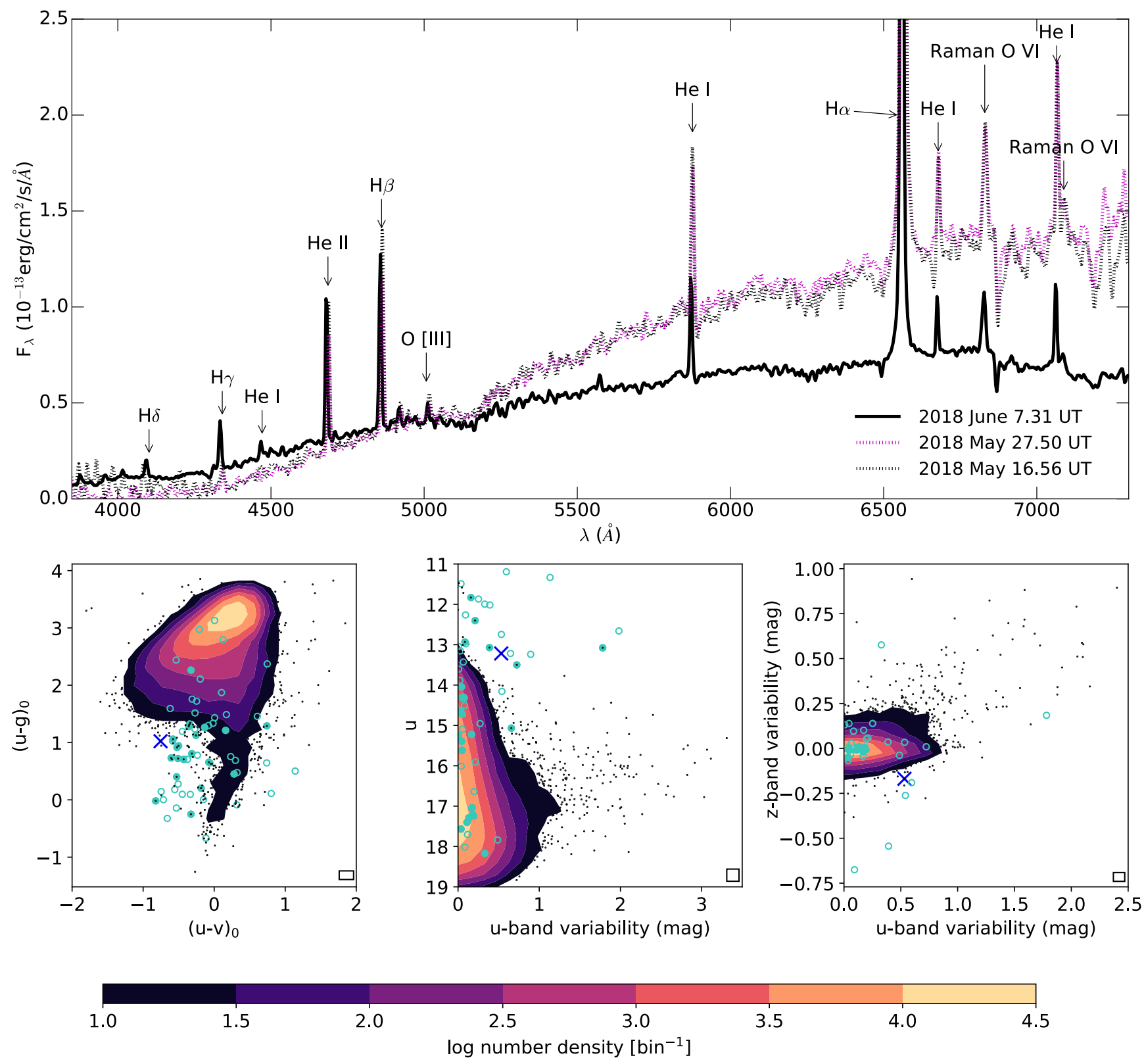

Figure 1. Upper panel: Our follow-up spectra of Hen 3-1768 exhibited the labeled emission lines. The first two were obtained with a 279mm Schmidt-Cassegrain telescope equipped with a LISA spectrograph (R 1500-1900, smoothed here to R 700; dotted lines) on 2018 May 16.56 and 27.50 UT and reduced in ISIS, the third at CASLEO with the REOSC spectrograph (R 700; solid line) on 2018 June 7.31 UT and reduced in IRAF. Flux calibrations are extremely preliminary, and absolute flux calibration was unavailable for the 2018 May 27.50 spectrum. Lower panels: We selected Hen 3-1768 (blue cross) from a sample of $\sim 210,000$ RGs (black points with contours over dense regions), because known symbiotics (turquoise circles, filled for symbiotics in the RG sample and hollow for symbiotics excluded from the RG sample by IR or quality cuts) are outliers in spaces defined by these parameters from SkyMapper data: $(\mathrm{u}-\mathrm{g})_{0},(\mathrm{u}-\mathrm{v})_{0}$, average $u$ magnitude, maximum $u$ variability between any two SkyMapper epochs, and $z$ variability between those same two epochs (available for only 90,000 RGs). The densest regions of parameter space are replaced by contours delineating the logarithmic number density of RGs (per bin; hollow rectangles). De-reddening was performed using total Galactic extinctions. Intervals between epochs range from 3 minutes to 1.5 years; the plotted interval for Hen 3-1768 is 142 days, larger than the sample median of 15 days. 
RG. H $\alpha$ emission was reported by the Henize (1976) survey. Hen 3-1768 was also observed by GALEX ${ }^{3}$, yielding a double-peaked spectral energy distribution in VizieR ${ }^{4}$.

Hen 3-1768 is one of 9 SkyMapper-selected stars whose symbiotic candidacies are supported by emission lines in follow-up optical spectroscopy. More recently, a 10th SkyMapper-selected candidate (GSC 09276-00130) was detected during performance tests of the RAMSES-II narrow-band Raman O VI filter (Angeloni et al., in preparation). Six of the 10, including Hen 3-1768, also meet new IR criteria for symbiotic candidacy independently developed by Akras et al. (2019, MNRAS, in press). We will investigate evidence for binary interaction in these candidates, along with a more complete set selected from the SkyMapper data, in a forthcoming paper.

We acknowledge the Gamilaroi people as the traditional owners of the land on which the SkyMapper Telescope stands. We acknowledge contributed spectra from F. Sims, F. Campos, M. Shara, and D. Zurek, and coordination by F. Teyssier (ARAS) and E. Waagen (AAVSO). A forthcoming paper will add additional acknowledgements. NSF DGE-1644869, AST-1616646

3 http://galex.stsci.edu/GR6/

${ }^{4}$ http://vizier.u-strasbg.fr/vizier/sed/ 


\section{REFERENCES}

Baella, N. O., Pereira, C. B., Miranda, L. F., et al. 2016, AJ, 151, 100.

Belczyński, K., Mikołajewska, J., Munari, U., et al. 2000, Astronomy and Astrophysics Supplement Series, 146, 407.

Bochanski, J. J., Willman, B., West, A. A., et al. 2014, AJ, $147,76$.

Gaia Collaboration, Brown, A. G. A., Vallenari, A., et al. 2018, A\&A, 616, A1.

Henize, K. G. 1976, ApJS, 30, 491.
Kunder, A., Kordopatis, G., Steinmetz, M., et al. 2017, AJ, 153,75 .

Li, J., Smith, M. C., Zhong, J., et al. 2016, ApJ, 823, 59.

Pickles, A. J. 1998, Publications of the Astronomical Society of the Pacific, 110, 863.

Shore, S. N., De Gennaro Aquino, I., Scaringi, S., et al. 2014, A\&A, 570, L4.

Skrutskie, M. F., Cutri, R. M., Stiening, R., et al. 2006, AJ, $131,1163$.

Wolf, C., Onken, C. A., Luvaul, L. C., et al. 2018, Publications of the Astronomical Society of Australia, 35, e010. 